\title{
Effects of Policosanol in Patients With Metabolic Syndrome: A Six-Month Study
}

\author{
Liuba Arteche-Hidalgo a, Julio Cesar Fernandez-Travieso ${ }^{\text {b, d }}$, Noysbel Suarez-Camejo ${ }^{\text {a }}$, \\ Juan Marin-Preval ${ }^{\mathrm{a}}$, Victor Alvarez-Acosta ${ }^{\mathrm{a}}$, Julian Chaviano-Pereira ${ }^{\mathrm{a}}$, \\ Maura Garcia-Sanchez ${ }^{a}$, Idelsis Esquivel-Moinelo ${ }^{a}$, Marisol Diaz-Gonzalez ${ }^{\mathrm{a}}$, \\ Odalys Matos-Reyes ${ }^{\mathrm{a}}$, Lilia Fernandez-Dorta ${ }^{\mathrm{b}}$, Jose Illnait-Ferrer ${ }^{\mathrm{b}}$, \\ Sarahi Mendoza-Castano ${ }^{\mathrm{b}}$, Maicel Monzon-Perez ${ }^{\mathrm{c}}$, Victoria Sanchez Pedroso ${ }^{\mathrm{c}}$
}

\begin{abstract}
Background: The metabolic syndrome comprises a set of cardiovascular risk factors represented by obesity of central distribution, dyslipidemias, glucose metabolism abnormalities and arterial hypertension, closely associated with insulin resistance. Policosanol is a mixture of high molecular weight alcohols purified from sugar cane wax with cholesterol-lowering and antioxidant effects. The aim of this study is to investigate in the medium term the effects of policosanol in patients with metabolic syndrome, as well as its safety and tolerability.
\end{abstract}

Methods: This phase IV study had a double-blind, randomized and controlled design, with two parallel groups that received policosanol $(10 \mathrm{mg} /$ day $)$ or placebo for 6 months. The study included patients with metabolic syndrome, of both sexes, aged between 25 and 70 years. As a primary efficacy variable, the effects on oxidative stress were evaluated, while the effects on lipids profile variables were considered as a secondary efficacy variable. Statistical analysis of the data was performed according to the intention-to-treat method.

Results: The study included 100 patients with metabolic syndrome ( 81 men, 19 women) (average age: 51 years). At the end of 6 months of treatment, policosanol significantly reduced the redox index with respect to the initial values and with respect to the placebo group. Policosanol significantly reduced levels of total cholesterol and lowdensity lipoprotein cholesterol (LDL-C), as well as increased serum levels of high-density lipoprotein cholesterol (HDL-C), while triglyceride levels although reduced at the end of treatment, this reduction was not significant. The policosanol was safe and well tolerate; it did not affect the physical and laboratory parameters investigated, with

Manuscript submitted April 20, 2020, accepted April 27, 2020

${ }^{a}$ Luis Diaz Soto Hospital, Havana, Cuba

bNational Centre for Scientific Research, Havana, Cuba

${ }^{\mathrm{c} N a t i o n a l ~ C l i n i c a l ~ T r i a l s ~ C o o r d i n a t o r ~ C e n t r e, ~ H a v a n a, ~ C u b a ~}$

${ }^{\mathrm{d} C o r r e s p o n d i n g ~ A u t h o r: ~ J u l i o ~ C e s a r ~ F e r n a n d e z-T r a v i e s o, ~ C l i n i c a l ~ T r i a l s ~ U n i t, ~}$ National Centre for Scientific Research, 25 Avenue and 158 St, Cubanacan, Playa, Havana, Cuba. Email: julio.fernandez@cnic.cu

doi: https://doi.org/10.14740/jem642 the exception of a significant and favorable reduction in the levels of apolipoprotein (Apo) B.

Conclusions: It is concluded that policosanol therapy for 6 months produces improvements on oxidative stress in patients with metabolic syndrome, in addition to a beneficial effect on their lipid profile, being safe and well tolerated.

Keywords: Policosanol; Metabolic syndrome; Oxidative stress; Redox index; Lipid profile

\section{Introduction}

The metabolic syndrome comprises a set of cardiovascular risk factors represented by obesity of central distribution, dyslipidemias, glucose metabolism abnormalities and arterial hypertension, closely associated with insulin resistance. It is considered as a predictor of cardiovascular morbidity and mortality, and a condition that predisposes to the future evolution of diabetes mellitus type $2[1]$.

It is not a single disease, but the association of health problems that can appear simultaneously or sequentially in the same individual. Its prevalence worldwide is increasing rapidly in recent years, with an estimated $25 \%$ of the world's adult population having metabolic syndrome [2-4].

Oxidative stress is related to various pathophysiological processes that occur in metabolic syndrome, both in its genesis and in the path of the atherogenic process. It occurs when the balance between antioxidant mechanisms and the production of pro-oxidant molecules is altered [5].

Several studies correlate the oxidative stress to the metabolic syndrome, and hold it responsible for important damages that occur in it, such as lipid peroxidation, damage to cell membranes, DNA damage, gene expression disorder and endothelial dysfunction, in addition to relating it with obesity, hypoadiponectinemia and cardiac, endothelial and renal disorders of metabolic syndrome $[6,7]$.

The first-line treatment of metabolic syndrome, both global and that of its individual components, is the modification 
of life habits aimed at losing weight and increasing physical activity. Thus, the Adult Treatment Panel III (ATP-III) highlights the need to intervene on lifestyle habits to prevent cardiovascular diseases in patients at higher risk, considering pharmacological treatment secondary, only indicated if there is a current recommendation on prevention of cardiovascular disease. However, some of the components of metabolic syndrome are very prevalent in different populations, regardless of their association with other factors in metabolic syndrome [8]. Therefore, up to $50 \%$ of patients with metabolic syndrome, in whom the weight reduction is satisfactory and who manage to maintain a desirable degree of physical activity, may need additional therapeutic measures for the adequate control of hypertension, diabetes or dyslipidemia $[9,10]$.

Policosanol is a mixture of high molecular weight alcohols purified from sugar cane (Saccharum officinarum, L) wax [11] with cholesterol-lowering effects due to the inhibition of cholesterol synthesis by regulating the activity of hydroxymethylglutaryl coenzyme (HMG CoA) through the increase of adenosine monophosphate (AMP) kinase activity [12-14].

The cholesterol-lowering effects of policosanol have been demonstrated in patients with type II hypercholesterolemia [11, 15-17]. Policosanol shows also relevant pleiotropic effects, such as the inhibition of platelet aggregation $[18,19]$ and the susceptibility of low-density lipoprotein (LDL) to be oxidised [20-22]. Clinical studies and long-term post marketing surveillance studies have proven that policosanol is safe and well tolerated [12-22, 23-25].

Taking into account the exposed background, it is logical to expect that policosanol will produce benefits on risk factors in patients with metabolic syndrome.

This study was undertaken to investigate the effects of policosanol administered for 6 months on oxidative stress and lipid profile in patients with metabolic syndrome, as well as safety and tolerability in this type of patients.

\section{Materials and Methods}

The study was conducted according to the principles reflected in the Helsinki statements (as revised in Brazil in 2013) [26], as well as the recommendations of the World Health Organization and the Cuban regulations on Good Clinical Practices. The study protocol was approved by the Ministry of Public Health and by the Ethics Committee in Clinical Research of the "Luis Diaz Soto" Hospital (IRB No. 2016-05-19), as well as registered in the Cuban Public Registry of Clinical Trials (RPCEC00000227).

\section{Study design}

This was a prospective, randomized, double-blinded, placebocontrolled study including 100 patients after randomization treated with placebo or policosanol for 6 months. The study was carried out in outpatient conditions and the sample was made up of patients with metabolic syndrome who attended a consultation at the "Luis Diaz Soto" Hospital, Havana, Cuba.

\section{Enrolment criteria}

Patients of both sexes, aged between 20 and 70 years, with evidence of metabolic syndrome who agreed to participate in the study after signing their informed consent were recruited. It is valid to note that in no case was the treatment of their diseases base prescribed to the patient by his doctors.

\section{Inclusion criteria}

Those patients who did not present any exclusion criteria and met the recruitment criteria with a diagnosis of metabolic syndrome according to NCEP-ATP III criteria [8], due to the presence of at least three of the following alterations: 1) Abdominal obesity: waist diameter in men $>102 \mathrm{~cm}$ and in women $>$ $88 \mathrm{~cm}$; 2) Blood pressure: values $\geq 130 / 85 \mathrm{~mm} \mathrm{Hg}$, or those patients who are receiving antihypertensive treatment; 3) Fasting triglycerides: blood values $\geq 1.70 \mathrm{mmol} / \mathrm{L}$; 4) Fasting high-density lipoprotein cholesterol (HDL-C): blood values $<$ $1.04 \mathrm{mmol} / \mathrm{L}$ in men and $<1.29 \mathrm{mmol} / \mathrm{L}$ in women; 5) Fasting glycemia: plasma values $\geq 5.6 \mathrm{mmol} / \mathrm{L}$ or that it has a prior and adequate diagnosis of diabetes mellitus or impaired glucose tolerance, whether or not it is under a therapeutic regimen of hypoglycemic medication or non-medication.

\section{Exclusion criteria}

Patients with history of ischemic or hemorrhagic vascular brain disease, major surgery in the 6 months prior to the start of the study, history of heart failure, history of liver failure, diagnosed neoplasms, alcoholism, psychiatric problems that limit their participation were excluded from this study; consumption of policosanol or other lipid-lowering as well as antioxidant or pro-oxidant medications in a period of 6 months prior to the study and usual medical history of allergy to medications or with any other special condition that at the doctor's discretion puts your health and life at risk during the study.

\section{Withdrawal criteria}

No desire to continue for any reason, any adverse event (AE) that required it (clinical or documented by complementary analyses), major violations of the protocol, such as lack of adherence to ingestion of treatment $>30$ consecutive days and/ or consumption of medications and/or supplements with lipidlowering and/or antioxidant action other than the study medication.

\section{Treatment}

Study medications were identical in appearance. The only difference was in the composition between policosanol and placebo tablets, since in the latter, $10 \mathrm{mg}$ of active ingredient was replaced by the same amount of lactose. Treatments 
were administered in identical packages identified by a code number and the number of treatment assigned by progressive inclusion. Study medications were randomised through a random allocation generated in the database center, consisting of balanced block of size 10, with a randomization ratio of 1:1. Tablets must be taken once a day with dinner for 6 months.

\section{Compliance assessment}

Compliance assessment were performed from visits three to six, compliance being assessed by patient questioning and tablet counts and defined as $\geq 85 \%$ of the scheduled tablets having been consumed since the prior visit.

\section{Concomitant medications}

During the study, simultaneous consumption of medications and/or supplements with hypolipidemic and/or antioxidant action other than the study medication was not allowed.

\section{Assessments}

Lipid profile and safety laboratory tests were performed at baseline and after 3 and 6 months of randomization. At each visit dietary reinforcement and physical examination were done.

\section{Efficacy analyses}

\section{Primary efficacy variables}

Significant reduction of oxidative stress and increased antioxidant defenses at the end of treatment with respect to the initial values and the placebo group.

\section{Secondary efficacy variables}

Significant reductions in triglyceride levels and/or a significant increase in HDL-C at the end of treatment with respect to initial values and placebo.

\section{Safety and tolerability analysis}

Data from physical examination (determination of bodyweight, body mass index, pulse rate and arterial pressure), laboratory tests (glucose, creatinine, aspartate aminotransferase, alanine aminotransferase, uric acid, urea, total proteins, alkaline phosphatase, apolipoprotein (Apo) A, Apo B) and requests for $\mathrm{AE}$ were included for safety and tolerability analysis. AEs were predefined as "mild" when did not require the withdrawal of study medication, and "moderate" when required therapy discontinuation according to doctors' criteria and/or specific treatment of the AEs, and "serious" when led to prolonged hospitalization, which could be fatal and non-fatal. Each AE was classified as having a causal relationship with treatment using the categories of definitely, probably, possibly, probably not, or definitively not drug-related [27].

\section{Laboratory analysis}

Blood samples were drawn after $12 \mathrm{~h}$ of overnight fasting in the Clinical Laboratory of the Luis Diaz Soto Hospital for processing and analysis. The variables of oxidative stress and antioxidant enzymes were determined according to established methods [28-31]. Lipid profile and laboratory test values were determined by enzymatic methods using reagent kits (Roche). Laboratory analyses were performed in a Hitachi 719 autoanalyzer. Determinations were done on the same sampling day. A quality control was performed throughout the study, so that precision (within and between day variations) and accuracy versus reference standards were controlled.

\section{Statistical analysis}

Statistical analysis for the whole study was planned in study protocol and amendments. All data were analyzed according to the intention-to-treat principle. Continuous values were compared using Student's $t$-test for paired (within group comparisons) and independent (between group comparisons) samples. Categorical data were compared with the $\chi^{2}$ test. All statistical tests were two-tailed, with significance at $\alpha=0.05$. Statistical analyses were performed using Statistics for Windows 10. Data management and statistical analysis were performed in the Department of Data Management and Processing of $\mathrm{Na}-$ tional Clinical Trials Coordinator Center.

\section{Results}

\section{Baseline patient characteristics}

Of the 115 patients recruited, 100 were eligible and randomized to policosanol $(n=50)$ or placebo $(n=50)$. The causes of non-inclusion were linked to the fact that in the complementary analyses indicated once recruited they presented normal laboratory values (triglycerides $<1.7 \mathrm{mmol} / \mathrm{L}$, HDL-C $>1.29$ $\mathrm{mmol} / \mathrm{L}$ and/or glucose $<5.6 \mathrm{mmol} / \mathrm{L}$ ).

Table 1 shows the main baseline characteristics of the study patients. Both groups were well matched at randomisation. The average age of the study population was 51 years and the distribution by sex showed a predominance of $81 / 100$ male patients $(81 \%)$ compared to $19 / 100(19 \%)$ of the female sex.

The patients included had other pathological antecedents, such as dyslipidemia (98\%), high blood pressure (95\%), obesity $\left(\geq 30 \mathrm{~kg} / \mathrm{m}^{2}\right)(50 \%)$, overweight $(43 \%)$, smoking $(23 \%)$, diabetes mellitus $(17 \%)$, thyroid disorders $(6 \%)$ and family history of coronary heart disease $(60 \%)$, diabetes $(45 \%)$, cerebrovascular disease (34\%) and peripheral disease (7\%). 
Table 1. Main Baseline Characteristics of Study Patients

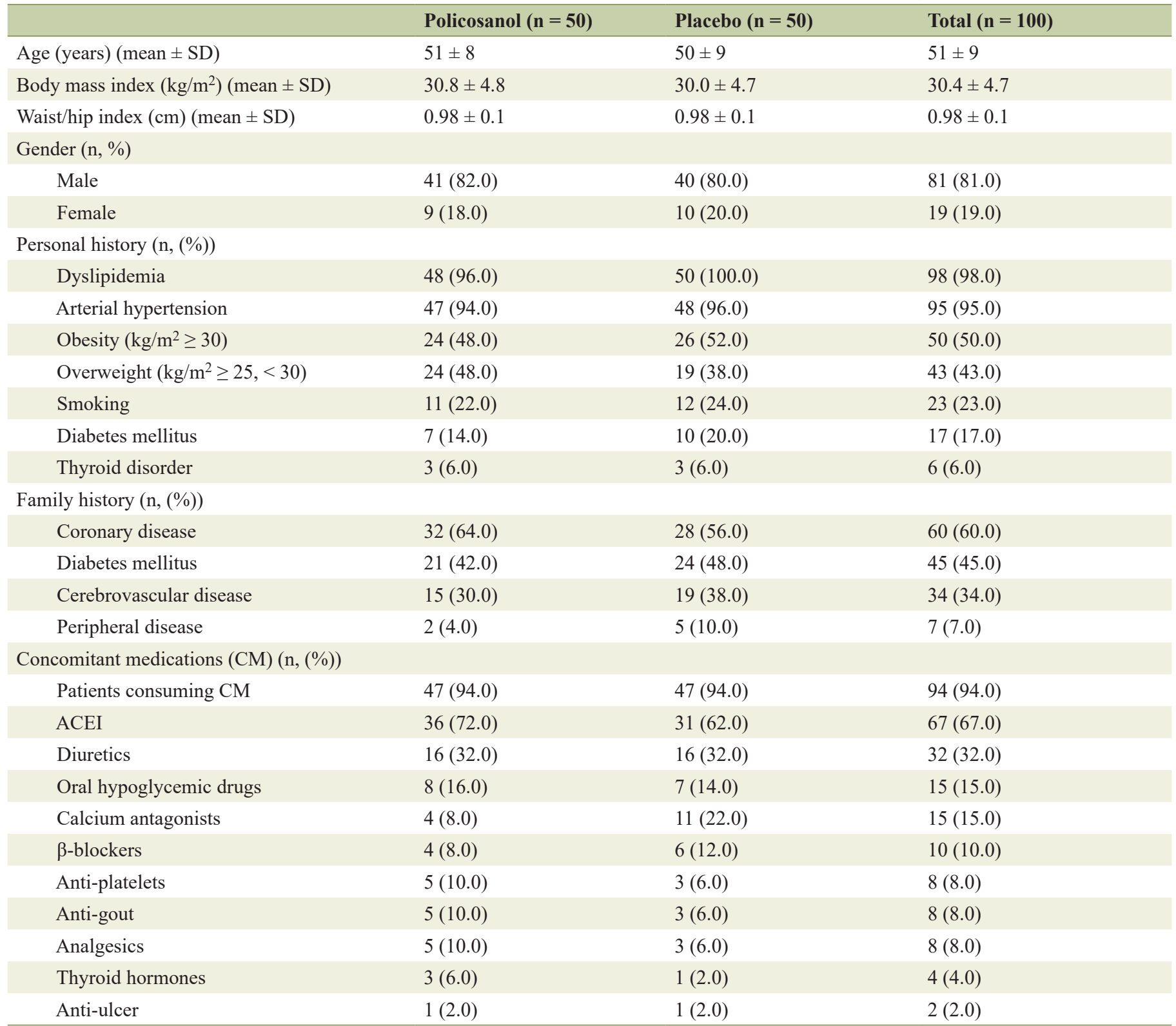

The table included concomitant medications consumed by $\geq 2$ patients. All comparisons were not significant (Student's $t$-test, $X^{2}$ test). SD: standard deviation; ACEl: angiotensin-converting enzyme inhibitor.

The frequency of consumption of concomitant therapy was high (94\%), similar in both groups, and in correspondence with their medical history, highlighting the consumption of antihypertensives (angiotensin-converting enzyme inhibitors (ACEIs), diuretics, calcium antagonists and $\beta$-blockers), followed by anti-diabetics, antiplatelet, anti-greases, analgesics, thyroid hormone treatment and antiulcer drugs.

Thirty-eight patients $(38 \%)$ caused withdrawal from the study: 34 due to protocol violation (the indicated complementary analyses were not performed or attended follow-up consultations), two for AEs, and two for trips (one abroad and another to provinces), without significant differences between groups.

With the exception of the 38 patients that caused discharge, the rest of the patients included consumed all the tablets scheduled for each stage according to the count of remaining tablets and questioning the patients, which shows an adequate adherence to the treatment that was similar in both groups.

\section{Effects on primary efficacy variables}

At the end of 6 months of treatment, policosanol significantly reduced $(\mathrm{P}<0.05)$ the redox index (main efficacy variable) by 
Table 2. Effects on Oxidative Values (Mean \pm SD)

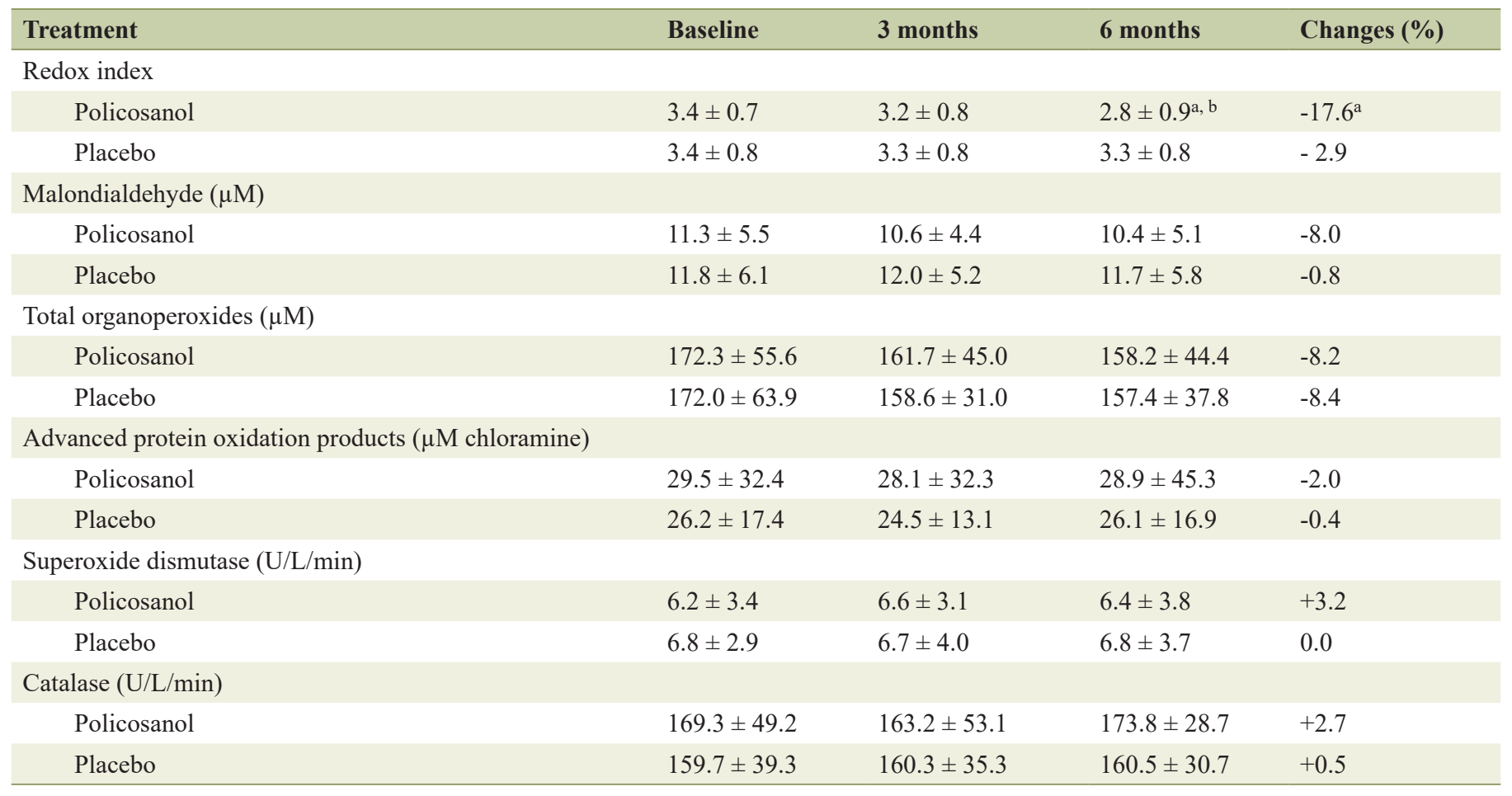

aP $<0.05$, comparison with placebo (Student's $t$-test for independent samples). ${ }^{\mathrm{b}} \mathrm{P}<0.01$, comparison with baseline (Student's $t$-test for paired samples). SD: standard deviation.

$17.6 \%$ with respect to initial values, and by $14.7 \%$ with respect to the placebo group (Table 2).

It also reduced, although not significantly, the levels of malondialdehyde, total organoperoxides and advanced protein oxidation products at the end of the treatment. Regarding the levels of antioxidant defenses (superoxide dismutase and catalase), although these were increased at the end of the treatment with policosanol versus baseline levels and the placebo group, this increase was not significant in the comparisons.

\section{Effects on secondary efficacy variables}

Treatment with policosanol increased HDL-C levels $(8.7 \%)$ (secondary efficacy variable) versus baseline, differences that were also significant in comparisons made with the placebo group, as well as significantly reduced serum low-density lipoprotein cholesterol (LDL-C) levels $(13.8 \%)$ and total cholesterol $(10.6 \%)$, while triglyceride values although reduced at the end of treatment, this reduction was not significant (Table 3).

\section{Safety and tolerability}

In the analysis of the effects on the physical indicators, no significant changes were obtained in any of the comparisons (data not shown in Table for simplicity).

In the analysis of the effects on the laboratory indicators investigated, only a significant decrease in Apo B values was observed in the group treated with policosanol with respect to baseline values and the placebo group in the comparisons (Table 4).

Two patients from the policosanol group reported AEs during the study; one classified as mild (decay) and another classified as severe, since the patient was hospitalized for 5 days for presenting a picture of loss of consciousness, hypoglycemia (glycemia: $2.5 \mathrm{mmol} / \mathrm{L})$, high blood pressure $(160 / 100 \mathrm{~mm}$ $\mathrm{Hg}$ ), preceded by a state of sustained stress. The patient during his hospitalization had neurological and cardiovascular studies with negative results, as well as daily glycemic profiles until his compensation, evolving satisfactorily. This event was also classified as doubtfully related to the treatment.

\section{Discussion}

The present study demonstrates that medium-term treatment (6 months) with policosanol (10 mg/day) in patients with metabolic syndrome produces an improvement in oxidative stress in these patients, in addition, to improve the lipid profile and be safe and well tolerated.

The average age of the patients included was 51 years, with a distribution by sex where male sex prevailed $(81 \%)$ compared to $19 \%$ female, which presented other pathological antecedents, such as dyslipidemia (98\%), hypertension arterial $(95 \%)$, obesity $\left(\geq 30 \mathrm{~kg} / \mathrm{m}^{2}\right)(50 \%)$, overweight $(43 \%)$, smoking $(23 \%)$, diabetes mellitus $(17 \%)$, thyroid disorders $(6 \%)$ 
Table 3. Effects on Lipid Profile (Mean \pm SD)

\begin{tabular}{|c|c|c|c|c|}
\hline Treatment & Baseline & 3 months & 6 months & Changes (\%) \\
\hline \multicolumn{5}{|c|}{ Total cholesterol (mmol/L) } \\
\hline Policosanol & $5.86 \pm 0.9$ & $5.26 \pm 0.9^{\mathrm{a}}$ & $5.24 \pm 0.8^{\mathrm{a}, \mathrm{b}}$ & $-10.6^{b}$ \\
\hline \multicolumn{5}{|l|}{ LDL-C (mmol/L) } \\
\hline Policosanol & $4.27 \pm 1.2$ & $4.12 \pm 1.1$ & $3.68 \pm 1.2^{\mathrm{a}, \mathrm{b}}$ & $-13.8^{b}$ \\
\hline Policosanol & $1.03 \pm 0.3$ & $1.08 \pm 0.2$ & $1.12 \pm 0.5^{\mathrm{a}, \mathrm{b}}$ & $+8.7^{b}$ \\
\hline Placebo & $1.10 \pm 0.5$ & $1.11 \pm 0.5$ & $1.05 \pm 0.3$ & -4.5 \\
\hline \multicolumn{5}{|c|}{ Triglycerides $(\mathrm{mmol} / \mathrm{L})$} \\
\hline Policosanol & $3.06 \pm 1.6$ & $2.89 \pm 1.4$ & $3.00 \pm 1.5$ & -2.0 \\
\hline
\end{tabular}

aP $<0.05$, comparison with baseline (Student's $t$-test for paired samples); ${ }^{\mathrm{P}} \mathrm{P}<0.05$, comparison with placebo (Student's $t$-test for independent samples). LDL-C: low-density lipoprotein cholesterol; HDL-C: high-density lipoprotein cholesterol; SD: standard deviation.

and family history of coronary heart disease $(60 \%)$, diabetes $(45 \%)$, cerebrovascular disease $(34 \%)$ and peripheral disease $(7 \%)$.

The consumption of concomitant medications was high (94\%) and well balanced in both groups. The most commonly used medications $(\geq 15 \%)$ were ACEI $(67 \%)$, diuretics $(32 \%)$, calcium antagonists $(15 \%)$, anti-diabetics $(15 \%)$, which is consistent with the number of hypertensive and diabetic patients included in the study.

Both groups were homogeneous in baseline conditions, as evidenced by the similarity of their demographic characteristics and the main response variables, which supports that the results obtained are attributable to the investigated treatment and not to disparity in the initial condition of the groups to be compared.

Thirty-eight patients (38\%) caused a withdrawal from the study, mainly due to a protocol violation (34\%), as they did not perform the complementary examinations and attend followup consultations, which we consider to be a limitation of the study that had a negative impact on obtaining more favorable results of the main efficacy variables investigated.

Treatment with policosanol (10 mg/day) for 6 months produced beneficial effects on the oxidative variables investigated, since it significantly reduced $(\mathrm{P}<0.05)$ the redox index (main efficacy variable) by $17.6 \%$ with respect to the initial values, and in $14.7 \%$ with respect to the placebo group. It also reduced, although not significantly, the levels of malondialdehyde, total organoperoxides and advanced protein oxidation products at the end of the treatment.

Regarding the levels of antioxidant defenses (superoxide dismutase and catalase), although these were increased at the end of the treatment with policosanol versus baseline levels and the placebo group, this increase was not significant in the comparisons.

These results obtained on the oxidative variables investigated are consistent with previous results obtained by policosanol in short- and medium-term studies conducted in other populations, in which their antioxidant effects were demonstrated [20-22].

If we take into account that oxidative stress is related to various pathophysiological processes that occur in the metabolic syndrome, both in its genesis and in the path of the atherogenic process and that in patients with metabolic syndrome, there is less protection against reactive species of oxygen and therefore, are exposed to the pathogenic effects of oxidative stress in vascular damage that leads to atherosclerosis, the antioxidant effects shown by policosanol in this study confirm its usefulness in the management of these patients [5-7].

On the other hand, in relation to the lipid profile, treatment with policosanol significantly increased levels of HDL-C (secondary efficacy variable) versus baseline and versus placebo. In addition, it significantly reduced serum LDL-C and total cholesterol levels versus baseline and versus placebo, while triglyceride values although decreased, were not significantly modified. However, we must point out that although policosanol showed an efficacy profile according to its effects on lipid variables (total cholesterol, LDL-C and HDL-C), these results were lower than those obtained in previous studies in other populations [15-22], but for the first time they are obtained in this type of patients.

Regarding the effects on triglycerides, these were modest, which coincides with previous studies in other populations where they have also been modest and inconsistent [15-22]. In addition, that the patients did not properly perform the indicated diet and the recommended exercise, which also had a negative impact on not obtaining more favorable results on this variable.

It was demonstrated that policosanol inhibits cholesterol synthesis in the first step of its metabolic pathway through activation of adenosine monophosphate protein kinase (AMPK), which in turn inhibit hydroxy-methyl-glutaryl-coenzyme A reductase [12-14]. AMPK, once activated, also inhibit acetyl CoA carboxylase (ACC). The inhibition of ACC increases fatty acid oxidation and reduces lipid synthesis, protecting in 
Table 4. Effects on Laboratory Indicators (Mean \pm SD)

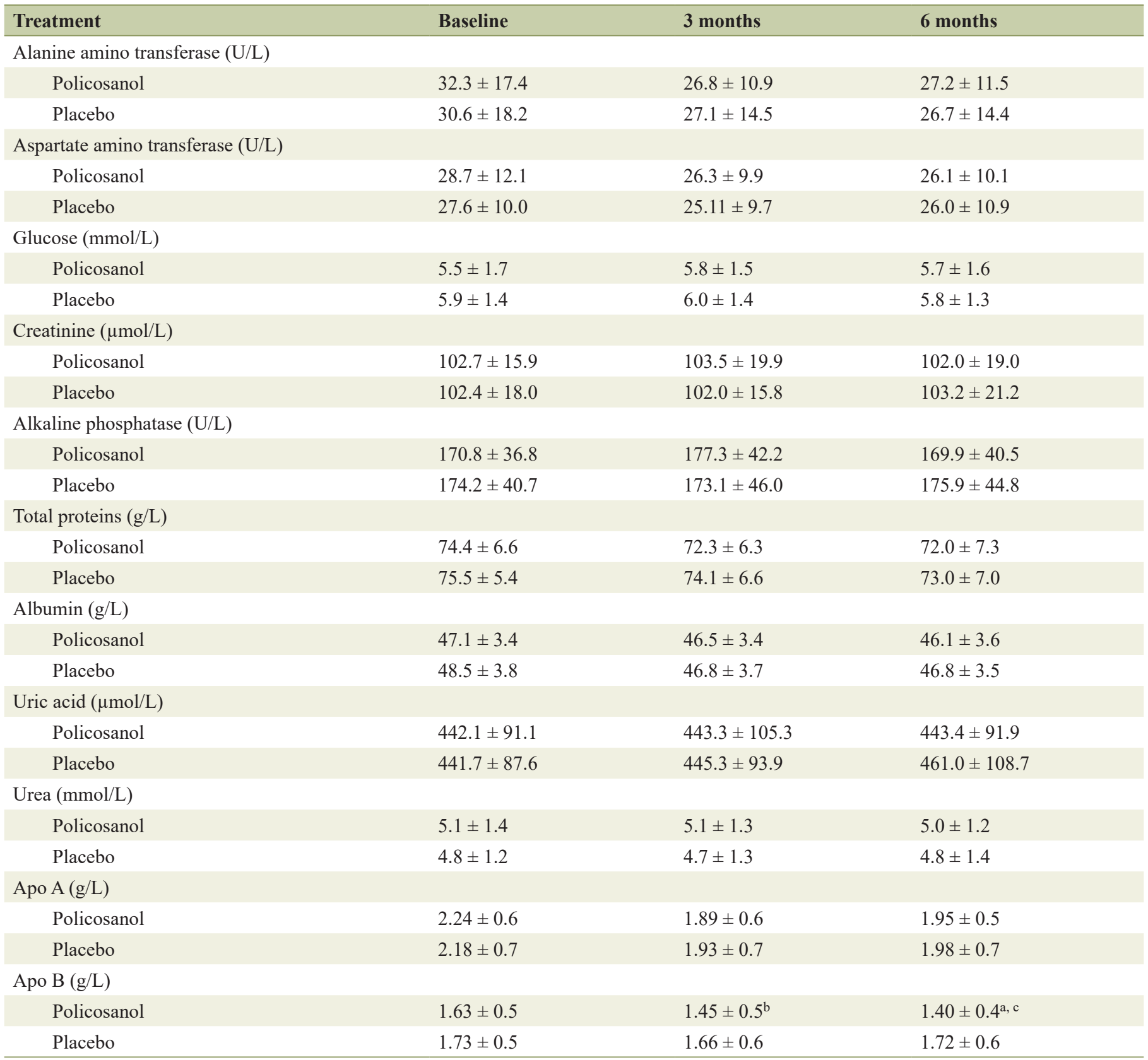

aP $<0.05$, comparison with baseline (Student's $t$-test for paired samples). ${ }^{\mathrm{b}} \mathrm{P}=0.052,{ }^{\mathrm{c} P}<0.01$, comparison with placebo (Student's $t$-test for independent samples). Apo: apolipoprotein; SD: standard deviation.

this way, muscle, heart, and others tissues from lipotoxicity. In addition, AMPK activation is associated with a wide array of beneficial effects, could explain the low level of side effect and compliance in the treated group versus placebo $[32,33]$.

After intestinal absorption, very long chain fatty alcohols (VLCFAs) are uptaken by the liver and partially converted into carboxylic acids. These results indicated that higher intake of VLCFA is significantly associated with favorable metabolic status including lower levels of circulating triglycerides. Other study confirmed that circulating serum VLCFAs were indepen- dently associated with favorable profiles of blood lipids (lower triglycerides and increase HDL-C); others cardiovascular disease risk markers and a lower cardiovascular disease risk by $52 \%[32,33]$.

On the other hand, fatty alcohols are substrates for the synthesis of plasmalogens in peroxisomes, which are potent endogenous antioxidants. Plasmalogens are released from the liver as component of lipoproteins thus protecting them from oxidation, and favoring its functionability.

Moreover, according with recent results, policosanol 
seems to present regeneration abilities via enhancement of HDL functionability [34, 35].

As expected, treatment with policosanol (10 mg/day) was safe and well tolerated, which coincides with previous data on the safety of policosanol in other populations and in clinical practice [15-25].

Regarding the parameters of the physical examination, there were no significant changes in the variables evaluated with respect to the baseline level and the placebo group. However, the fact that the body weight and waist/hip index of the patients remained without significant changes at the end of the study, indicates that the patients did not properly perform the recommended diet and exercise, which constitutes another limitation in this study, taking into account the high number of patients $(93 \%)$ included with overweight and obesity, risk factors for the metabolic syndrome that were not objectively modified.

Regarding the parameters of blood biochemistry, the treatment with policosanol did not produce modifications of the investigated variables, with the exception of a significant favorable reduction of Apo B concentrations at 6 months of treatment with respect to baseline levels and the placebo group, which corresponds to the significant reduction of the LDL-C levels obtained in this group.

Taking into account the significant increase in HDL-C levels, an increase in the concentration of Apo A should also be expected. However, contrary to expectations, the concentration of Apo A decreased, which can be interpreted as that the HDL with a lower amount of Apo A managed to transport more cholesterol, due to an increase in its functionality with respect to its capacity in the uptake of cholesterol for reverse transport.

Only two patients in the policosanol group reported AEs during the study, one classified as mild (decay) and another classified as severe due to hospitalization. However, this event was also classified as doubtfully related to the treatment.

It is concluded that policosanol $(10 \mathrm{mg} /$ day $)$ for 6 months produces improvements on oxidative stress in patients with metabolic syndrome, in addition to a beneficial effect on their lipid profile, being safe and well tolerated. Future studies with a larger sample size and longer treatment time should confirm these results in this type of patients.

\section{Acknowledgments}

None to declare.

\section{Financial Disclosure}

This study was support by the National Centre for Scientific Research, as part of its research-development projects.

\section{Conflict of Interest}

The authors declare that there is no conflict of interest in relation to this work.

\section{Informed Consent}

Informed consents were obtained from the patients.

\section{Author Contributions}

The authors have been involved in the conception and design of the work, or in the collection of data, or in the analysis and interpretation of the data, as well as in the writing of the article or in its critical review and in the approval of the final version for publication.

\section{Data Availability}

The authors declare that data supporting the findings of this study are available within the article, and any inquiries regarding supporting data availability of this study should be directed to the corresponding author.

\section{References}

1. Aguilar-Salinas CA, Viveros-Ruiz T. Recent advances in managing/understanding the metabolic syndrome. F1000Res. 2019;8(F1000 Faculty Rev):370.

2. Dommermuth R, Ewing K. Metabolic syndrome: systems thinking in heart disease. Prim Care. 2018;45(1):109-129.

3. Lizazaburi J. Sindrome metabolico: concepto y aplicacion practica. An Fac Med. 2013;74(4):315-320.

4. Grundy SM. Metabolic syndrome update. Trends Cardiovasc Med. 2016;26(4):364-373.

5. Carrier A. Metabolic syndrome and oxidative stress: a complex relationship. Antioxid Redox Signal. 2017;26(9):429-431.

6. Spahis S, Borys JM, Levy E. Metabolic Syndrome as a multifaceted risk factor for oxidative stress. Antioxid Redox Signal. 2017;26(9):445-461.

7. Bonomini F, Rodella LF, Rezzani R. Metabolic syndrome, aging and involvement of oxidative stress. Aging Dis. 2015;6(2):109-120.

8. Expert panel on detection evaluation, treatment of high blood cholesterol in adults. Executive summary of the third report of the National Cholesterol Education Program (NCEP) expert panel on detection, evaluation, and treatment of high blood cholesterol in adults (Adult Treatment Panel III). JAMA. 2001;285(19):2486-2497.

9. Vona R, Gambardella L, Cittadini C, Straface E, Pietraforte D. Biomarkers of oxidative stress in metabolic syndrome and associated diseases. Oxid Med Cell Longev. 2019;2019:8267234.

10. Rani V, Deep G, Singh RK, Palle K, Yadav UC. Oxidative stress and metabolic disorders: Pathogenesis and therapeutic strategies. Life Sci. 2016;148:183-193.

11. Mas R. Policosanol. Drugs of the Future. 2000;25:569586.

12. Singh DK, Li L, Porter TD. Policosanol inhibits choles- 
terol synthesis in hepatoma cells by activation of AMPkinases. J Pharmacol Exp Ther. 2006;106:107-144.

13. Oliaro-Bosso S, Calcio Gaudino E, Mantegna S, Giraudo E, Meda C, Viola F, Cravotto G. Regulation of HMGCoA reductase activity by policosanol and octacosadienol, a new synthetic analogue of octacosanol. Lipids. 2009;44(10):907-916.

14. Banerjee S, Porter TD. Tea and policosanol act through different mechanisms to activate AMP-kinase and suppress HMG-CoA reductase to inhibit cholesterol synthesis. Proceedings of the FASEB meeting. FASEB J. 2010;24(Suppl 1):541.23.

15. Aneiros E, Mas R, Calderon B, Illnait J, Fernandez L, Castano G, Fernandez JC. Effect of policosanol in lowering-cholesterol levels in patients with type II hypercholesterolemia. Curr Ther Res Clin \& Exptl. 1995;56:176182.

16. Mas R, Castano G, Illnait J, Fernandez L, Fernandez J, Aleman C, Pontigas V, et al. Effects of policosanol in patients with type II hypercholesterolemia and additional coronary risk factors. Clin Pharmacol Ther. 1999;65(4):439-447.

17. Canetti M, Morera MS, Mas R, Illnait J, Fernandez L, Fernandez JC. Effects of policosanol on primary hypercholesterolemia: A 3-year open follow-up. Curr Ther Res Clin \& Exptl. 1997;58:868-875.

18. Arruzazabala ML, Mas R, Molina V, Carbajal D, Mendoza S, Fernandez L, Valdes S. Effect of policosanol on platelet aggregation in type II hypercholesterolemic patients. Int J Tissue React. 1998;20(4):119-124.

19. Castano G, Mas R, Arruzazabala ML, Noa M, Illnait J, Fernandez JC, Molina V, et al. Effects of policosanol and pravastatin on lipid profile, platelet aggregation and endothelemia in older hypercholesterolemic patients. Int J Clin Pharmacol Res. 1999;19(4):105-116.

20. Menendez R, Mas R, Amor AM, Gonzalez RM, Fernandez JC, Rodeiro I, Zayas M, et al. Effects of policosanol treatment on the susceptibility of low density lipoprotein (LDL) isolated from healthy volunteers to oxidative modification in vitro. Br J Clin Pharmacol. 2000;50(3):255262.

21. Menendez R, Mas R, Amor A, et al. Effects of policosanol on the low density lipoprotein (LDL) isolated on hypercholesterolemic patients at high coronary risk to in vitro copper-mediated lipid peroxidation. A randomised, double-blinded pilot study. Curr Ther Res Clin \& Exptl. 2000;61:609-620.

22. Castano G, Menendez R, Mas R, Amor A, Fernandez JL, Gonzalez RL, Lezcay M, et al. Effects of policosanol and lovastatin on lipid profile and lipid peroxidation in patients with dyslipidemia associated with type 2 diabetes mellitus. Int J Clin Pharmacol Res. 2002;22(3-4):89-99.

23. Fernandez L, Mas R, Illnait J, Fernandez JC. Policosanol: results of a postmarketing surveillance control on 27879 cases. Curr Ther Res. 1998;59:717-722.

24. Mas R, Rivas P, Izquierdo JE, Hernandez R, Fernandez L, Fernandez J, Orta SD, et al. Pharmacoepidemiologic study of policosanol. Curr Ther Res. 1999;60:458-467.

25. Fernandez S, Mas R, Gamez R, Diaz A, Fernandez J, Orta $\mathrm{SD}$, Illnait J, et al. A pharmacological survillance of policosanol tolerability in the elderly. Am J Gerontol Pharmacother. 2004;2:11-16.

26. World Medical Association. Declaration of HelsinkiEthical principles for medical research involving human subjects (Revised in Brazil, 2013).

27. Requerimientos para la notificacion y el reporte de eventos adversos graves e inesperados en los ensayos clinicos. Regulacion No. 45-2007, Centro para el Control Estatal de los Medicamentos, Equipos y Dispositivos Medicos (CECMED), MINSAP, La Habana, Cuba, 2007.

28. Esterbauer H, Cheeseman KH. Determination of aldehydic lipid peroxidation products: malonaldehyde and 4-hydroxynonenal. Methods Enzymol. 1990;186:407-421.

29. Witko-Sarsat V, Friedlander M, Nguyen Khoa T, Capeillere-Blandin C, Nguyen AT, Canteloup S, Dayer JM, et al. Advanced oxidation protein products as novel mediators of inflammation and monocyte activation in chronic renal failure. J Immunol. 1998;161(5):2524-2532.

30. SIGMA. Biochemical organic compounds. Diagnostic Reagents; 1993.

31. Boehringer Mannheim. Biochemical information. A revised biochemical reference sources. Enzymes for routine (1st edition), Germany. Boehringer Mannheim. 1987; p. 15-16 and 80-81.

32. Cho KH, Bae MA, Kim JR. Cuban sugar cane wax acid and policosanol showed similar atheroprotective effects with inhibition of LDL oxidation and cholesteryl ester transfer via enhancement of high-density lipoproteins functionality. Cardiovasc Ther. 2019;2019:8496409.

33. Cho KH, Kim SJ, Yadav D, Kim JY, Kim JR. Consumption of Cuban policosanol improves blood pressure and lipid profile via enhancement of HDL functionality in healthy women subjects: randomized, double-blinded, and placebo-controlled study. Oxid Med Cell Longev. 2018;2018:4809525.

34. Lee EY, Yoo JA, Lim SM, Cho KH. Anti-aging and tissue regeneration ability of policosanol along with lipid-lowering effect in hyperlipidemic zebrafish via enhancement of high-density lipoprotein functionality. Rejuvenation Res. 2016;19(2):149-158.

35. Kim JY, Kim SM, Kim SJ, Lee EY, Kim JR, Cho KH. Consumption of policosanol enhances HDL functionality via CETP inhibition and reduces blood pressure and visceral fat in young and middle-aged subjects. Int J Mol Med. 2017;39(4):889-899. 